\title{
Prevalence, Comparison, and Determinants of Smoking among Young Schoolchildren in Kingdom of Saudi Arabia
}

\author{
Khalid Ansari ${ }^{1}$, Faraz Ahmed Farooqi ${ }^{2}$, Syed Taha Abidi ${ }^{3}$, Jaffar Shawqi Al Mubarak ${ }^{4}$, Saqib Ali $^{5 *}$ \\ ${ }^{1}$ Department of Respiratory Care, College of Applied Medical Science, Imam Abdulrahman Bin Faisal University, Dammam, \\ Saudi Arabia; ${ }^{2}$ Department of Academic Affairs, College of Dentistry, Imam Abdulrahman Bin Faisal University, Dammam, \\ Kingdom of Saudi Arabia; ${ }^{3}$ Department of Public Health, Saudi Electronic University, Kingdom of Saudi Arabia; ${ }^{4}$ Department of \\ Respiratory Care, Imam Abdulrahman Bin Faisal University, Dammam Kingdom of Saudi Arabia; ${ }^{5}$ Department of Biomedical \\ Dental Sciences, College of Dentistry, Imam Abdulrahman Bin Faisal University, Dammam, Saudi Arabia
}

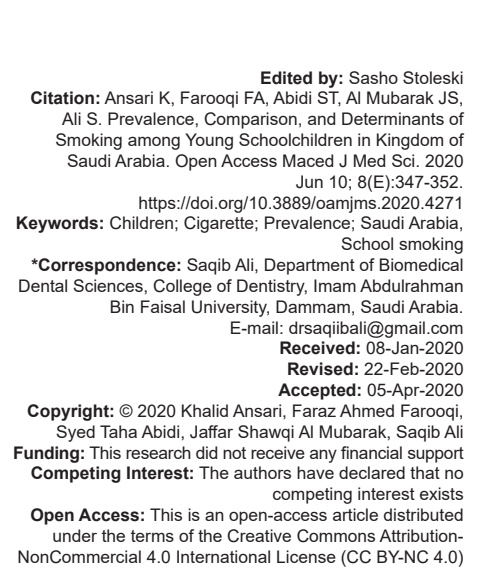

\section{Introduction}

Smoking is one of the foremost causes of preventable death. The World Health Organization stated tobacco use currently being responsible for the death of one in 10 adults' globally estimating around 5 million deaths per year. Moreover, unless the circumstances change, in coming 25 years, the predicted death toll will almost double; millions of people more will prematurely develop tobacco-related illnesses, leading to chronic disability [1], [2].

In Saudi Arabia, this is becoming a serious health issue among all age groups, particularly in children [3], [4], [5]. Tobacco smoking in children is considerably increased among 16-18 years adults [6], [7]. Almost 3900 children (0.02\%) under 18 years of age try their first cigarette on daily basis. Of those $25 \%$ become continuous smokers and $50 \%$ get addicted and/or die with chronic lung diseases [8], [9]. Furthermore, the previous study also suggests that people who start smoking in the early ages are more likely to develop addiction to nicotine than those who start smoking at an older age [10], [11]. In the UK, two-thirds of adult smokers stated that they took up smoking before 18 years of age and $80 \%$ before the age of 20 [3]. In 2011, the overall status of current smoking among Australian students age 12-17 years was $6.7 \%$, and among $12-15$ years old, $4.1 \%$ were current smokers [12]. More studies are necessary to examine the prevalence and earliest age of smoking in children in the Saudi society and the present study will explore this area further to find the earliest age of smoking among our cohort of schoolchildren.

The previous study also suggests that among ages $10-19$ years, $47.7 \%$ started to smoke at the age of 16 years or earlier with higher proportion of males with the mean age at the time of first cigarette was 6-13 years [11]. This study will further explore this phenomenon of earliest age of smoking among Saudi schoolchildren.

According to the authors understanding, this study is the first to estimate the prevalence, understand the determinants of tobacco use from public as well as private schools in Dammam, Saudi Arabia. In addition, smoking behavior comparison and the earliest age 
of exposure to smoking among schoolchildren were explored.

\section{Materials and Methods}

This cross-sectional study was conducted in the public and private schoolchildren in the city of Dammam, Saudi Arabia. Ethical approval was attained and permission was taken from school administration. Following that, a letter was sent to the children's parents with the information sheet about the research, questionnaire used, and consent form 1 day before the start of the study. A total of 866 male students were recruited. The sample size was calculated through stratified random sampling. The inclusion criteria were male intermediate students from Eastern Province. Only those students who have signed a consent form were authorized to participate. Students not belonging to Eastern Province and non-Saudi were excluded from the study.

The modified version of a standard, structured, and validated Global Youth Tobacco Survey (GYTS) questionnaire was used [13], [14]. The modification was made for three reasons. First, to make it a little shorter so that our study population does not get exhausted while filling the form as the original comprehensive version consists of 56 core questions [14]. Second, because some questions were not suitable to ask considering culture of Saudi and/or to avoid any chance of biased responses. Furthermore, to add more clarity to the questionnaire, it was divided into seven separate sections; however, the questions and sections followed the same sequence as of GYTS. To further test the students' understanding of the questions, the research team conducted a focus group discussion with students aged 10-15 years. The seven sections are as follows: Use of tobacco, feelings toward stopping smoking, exposure to other smokers, getting cigarettes, knowledge of message that is against using tobacco, knowledge of advertisement or promotion for tobacco, and attitudes and beliefs about using tobacco.

The team was educated to make sure a unified method of data collection and to encourage students to answer all the questions without being in stress during data collection process. The data were collected by the research team members in the absence of any teacher or school supervisor, so the students could feel free while responding to the questions. Centers for disease control and prevention (CDC) guidance was followed during data collection. Survey was explained to each class. Participants were informed of their information confidentiality and that they had the right to refuse participation in the survey. They were also asked that each question be responded independently without taking any help from fellow students. A total of 25 min were initially given to the students as permitted by the school administration as it is considered sufficient to respond all the questions. The questionnaires were collected after the allotted time. However, extra 5 min were given to those who were unable to complete the questionnaire to ensure students comfort while filling the form and to avoid any bias responses.

The major outcome variable is smoking, referred to as cigarette smoking only. The smoking status or current smoking in the past few days to 4 weeks was determined using a questionnaire. Another important variable was public and private schools, the questionnaire was pre-coded, and this was determined by random selection of private and public schools that consented to participate in this study. Other variables included were sociodemographic data (age, gender, parent's educational qualification, marital status, profession, living status, whether any of students relatives smoke, or use any other form of other tobacco, [other than cigarettes], and when students smoked their first cigarette).

\section{Statistical analysis}

Data were analyzed using SPSS (Version 22, IBM Corporation). Descriptive results for demographic information are presented in Table 1. Chi-square was used to determine the association between smokers and factors that are associated with smoking among the adolescents for categorical data (These include type of schooling, parents smoking history, and parents working status) and for quantitative data, the independent sample t-test was used. A step-wise logistic regression was used to determine the risk factors for current smoking. Before doing logistics regression, the factor analysis (principal component analysis) was performed to produce the factors and among those factors, the variables with larger factor loading were selected for step-wise logistic regression. To assess the goodness of fit of the

Table 1: Demographics results of the study population

\begin{tabular}{ll}
\hline Variables & $(\mathrm{n}, \%),($ Mean \pm SD $)$ \\
\hline Age & $866(100),(14.20 \pm 1.03)$ \\
Type of school & $643(74.2)$ \\
Public & $223(25.7)$ \\
Private & $233(26.9)$ \\
Level (study year) & $258(29.8)$ \\
$1^{\text {st }}$ & $375(43.3)$ \\
$2^{\text {nd }}$ & \\
$3^{\text {rd }}$ & $622(71.7)$ \\
Smoking status & $241(27.8)$ \\
Never smoked & \\
Tried to smoke & $391(45.1)$ \\
Father's education & $220(25.4)$ \\
Professional & $225(29.4)$ \\
Non-professional & \\
Unknown & $286(33)$ \\
Mother's education & $237(27.3)$ \\
Professional & $343(39.6)$ \\
Non-professional & \\
Unknown & $511(58.89)$ \\
Relative smoking & $355(40.87)$ \\
Smokers & \\
Non-smokers & $829(96)$ \\
Living status & $17(2)$ \\
Family & $12(1.4)$ \\
Father only & $4(0.5)$ \\
Mother only & \\
Others & \\
\hline
\end{tabular}


model, Hosmer-Lemeshow statistics was used [15]. Missing information was filled with the mode of same variable instead of deleting. Odds ratios (ORs) and 95\% confidence intervals were obtained for each variable as an estimate of risk factor of current smoking. All analyses were considered as significant at an alpha level of 0.05 .

\section{Results}

\section{Overall}

A questionnaire was administrated in 1012 students and a total of 866 (response rate $85.50 \%$ ) students participated in the survey. About $4.50 \%$ were absent on the day of data collection and $10 \%$ did not bring the consent form to participate in the survey.

The results revealed that $246(27.8 \%)$ of children tried to smoke in their life. The demographic characteristics of the study subjects are presented in Table 1. The mean age of the study participants was $14.20 \pm 1.03$ years. About $74 \%$ (643) recruited from public and $26 \%(n=223)$ were from private schools. The data about parent's education, relative smokers, and living status are also presented in Table 1.

\section{Students Behavior about smoking}

\section{Age}

The distribution of onset of smoking among schoolchildren in different age groups showed that the earliest age when children start trying to cigarette smoke is 7 years or younger $(3 \%)$, and the most common age when students start trying to smoke is between 10 and 13 years $(12 \%)$. No age difference was found between smoker (14.44 \pm 1.09$)$ and non-smoker (13.99 \pm 0.98) ( $p>0.05)$.

\section{Relative smoker}

Table 2 indicates that the students who have a greater number of relative smokers have significantly a higher prevalence of smoking (36\%) than those with no relative smoking $(15 \%)(p<0.05)$.

Table 2: Relationship between relative's smoking status and students smoking habit

\begin{tabular}{llll}
\hline Relative smoking status & \multicolumn{2}{l}{ Students smoking habit } & \multirow{2}{*}{-value } \\
\cline { 2 - 3 } & Never smoked $\mathrm{n}(\%)$ & Tried to smoke $\mathrm{n}(\%)$ & \\
\hline Smokes (508) & $323(63.58)$ & $185(36.41)$ & ${ }^{*} \mathrm{p}=0.001$ \\
Does not smoke (354) & $299(34.46)$ & $55(15.53)$ & \\
\hline${ }^{*}$ Significant at $\mathrm{p}<0.05$ (Chi-square test). & &
\end{tabular}

\section{Quitting smoking}

Table 3 shows that $40 \%$ of students have a feeling toward quitting smoking and they have support from their friends and family. They are also getting knowledge about the harmfulness of smoking and warning messages from anti-tobacco campaigns. In addition, some students' relatives are aware of their smoking but did not try to stop or convince them not to smoke.

Table 3: Feeling to quit smoking and knowledge from antitobacco messages

\begin{tabular}{|c|c|c|c|}
\hline Statements & Yes (\%) & No $(\%)$ & Unsure (\%) \\
\hline Do you want to stop smoking? & 60 & 30 & 10 \\
\hline Did you tried to stop in the past 12 months? & 59.2 & 40.8 & 0 \\
\hline $\begin{array}{l}\text { Have you received any help from friends or } \\
\text { professionals? }\end{array}$ & 57.6 & 32.4 & 10 \\
\hline Does any of your relative asked you to stop? & 37.6 & 47.2 & 15.2 \\
\hline Did you see any anti-tobacco message on social media? & 37.6 & 40 & 22.4 \\
\hline $\begin{array}{l}\text { During the past } 12 \text { months anybody taught you } \\
\text { dangerous of tobacco? }\end{array}$ & 46.4 & 41.6 & 12 \\
\hline
\end{tabular}

\section{Factors to promote smoking}

Table 4 describes the comparison and possible factors for students to smoke or to try to smoke. When compared, we found that smoking inside the private schools $(60 \%)$ is higher than public schools $(54 \%)$. Overall prevalence of smoking was found higher in public schools.

Table 4: The comparison and possible factors for students to smoke or tried to smoke

\begin{tabular}{lllllll}
\hline Factors & \multicolumn{3}{l}{ Public school } & & \multicolumn{2}{l}{ Private school } \\
\cline { 2 - 3 } & Yes (\%) & No (\%) & & Yes (\%) & No (\%) & p-value \\
\hline Family smoking & 60.06 & 39.94 & & 56.05 & 43.95 & 0.294 \\
Education level of family & 57.08 & 42.92 & & 84.87 & 15.13 & $0.000^{*}$ \\
Help or advice to stop smoking & 26.22 & 73.78 & & 25.56 & 74.44 & 0.498 \\
Smoke inside school & 54.77 & 45.23 & & 60.09 & 39.91 & 0.250 \\
Other's smoke is harmful & 80.16 & 19.84 & & 75.23 & 24.77 & 0.309 \\
Availability of tobacco & 31.71 & 68.29 & & 84.75 & 15.25 & $0.001^{*}$ \\
\hline "Significant at p<0.05 (Chi-square test). & & & & & &
\end{tabular}

Knowledge about the harmfulness of smoke from other smoker is higher $(80 \%)$ in public schools. This study also found significant difference between public and private schools in terms of their availability of tobacco (85\% vs. 32\%; Chi-square, $p=0.001$ ).

The study also attempted to explore the factors that made children smoke. Only $15.13 \%$ of the population tried to smoke due to the feeling of being more comfortable when they smoke $(p=0.001)$. There is no significant association with the father's education and a smoking child ( $p=0.054$ ) but the mother's education has a significant association with a student's smoking habit ( $p=0.033)$.

Logistics regression analysis reveals that growing age and getting cigarettes form someone are significantly associated with the current smoking $([\mathrm{OR}=2.08],[\mathrm{OR}=2.16]$, respectively $)$. HosmerLemeshow test $(p=0.002)$ confirms the best fitting of model.

In addition, the desire to continue smoking in next 12 months was strongly correlated with current smoker (OR 1.68). Perceiving that smoking helps people to feel more comfortable during the parties is a strong risk factor of smoking (OR 1.36). The availability of cigarette for free (from friends or other 
sources) is significantly associated with the risk of smoking (OR 1.51, OR 1.2, respectively). The risk is increased among the current smokers, having smoker parents, and smoking inside the house $(\mathrm{OR}=1.16$, OR =1.06). Interestingly, only the father's education level $(O R=0.92)$ and type of studying $(O R=0.96)$ schools are weakly associated with the smoking. The perspective of non-smokers (considered themselves as passive smokers) were that the inhalation of smoke is more dangerous than smoking tobacco itself. Watching smoking scenes in movies also had weak association with the smokers (Table 5).

Table 5: Logistics regression analysis of smoking risk factors

\begin{tabular}{lllll}
\hline Factors & $\mathrm{B}$ & $\mathrm{S} . \mathrm{E}$. & $\mathrm{OR}$ & $95 \% \mathrm{Cl}$ \\
\hline Age & 0.731 & 0.178 & $2.08^{*}$ & $1.466-2.947$ \\
Type of school & -0.045 & 0.264 & 0.960 & $0.57-1.605$ \\
Living with parents & 0.204 & 0.314 & 1.230 & $0.662-2.269$ \\
Father education & -0.089 & 0.252 & 0.920 & $0.558-1.5$ \\
Smoking inside home & 0.089 & 0.071 & 1.090 & $0.952-1.255$ \\
Smoker parents & 0.150 & 0.079 & $1.16^{*}$ & $0.996-1.356$ \\
Smoked inside school in presence & 0.105 & 0.220 & 1.110 & $0.722-1.708$ \\
Getting cigarettes easily & 0.770 & 0.178 & $2.16^{*}$ & $1.523-3.06$ \\
Smoking in movies & -0.076 & 0.230 & 0.99 & $0.59-1.454$ \\
Offered free tobacco & 0.227 & 0.664 & 1.250 & $0.342-4.61$ \\
Tobacco offered by friend & 0.410 & 0.191 & $1.51^{*}$ & $1.037-2.19$ \\
Use any tobacco in next 12 months & 0.522 & 0.190 & $1.68^{*}$ & $1.162-2.444$ \\
Smoking help people feel more comfortable & 0.306 & 0.105 & $1.36^{*}$ & $1.104-1.669$ \\
\hline${ }^{*}$ Significant at 0.05, B: Coefficient of models, S.E: Standard error, OR: Odds ratio, Cl= Confidence interval.
\end{tabular}

\section{Discussion}

According to the authors understanding, this is the first study which attempted to estimate the prevalence of smoking among Saudi male children with comparing public and private schools along with its determinants. This study also attempted to explore at what point the students typically begin to smoke cigarettes.

The results of this study suggest that overall $27.8 \%$ of children tried to smoke in their early life which is alarming as these children are at a high risk of becoming cigarette smokers in the future. The findings are consistent with similar studies reporting that more than 2800 kids in the USA try their first cigarette every day and 700 kids under 18 years become new regular, daily smokers [16], [17]. The results also revealed that $71.74 \%$ being a high percentage of students who never tried to smoke, but they tried to smoke at an early age of 7 years or younger (3\%) being consistent with a previous finding [16].

Our study results also indicated that the most common age when students start trying to smoke is between 10 and 13 years (12\%) followed by 14 and 16 years $(8.3 \%)$. These findings are supported by studies showing that each day more than 3800 youth, aged 18 years or younger, smoke their first cigarette, and an additional 2100 youth and young adults become daily cigarette smokers [18], [19]. The present study also investigated student's behavior for inclination toward smoking and its prevalence. The results from logistics regression revealed that age, peer smokers, and wrong perception that smoking gives comfortable feelings, easy access to cigarettes, smoking inside the house, and offered from close friends have a strong correlation with smoking. These findings show similarity to previous published results of adolescent looking up to their parents and relatives for replicating their actions and supplements in development of smoking habit [20], [21]. The results also showed $36.41 \%$ of students tried to smoke with relatives in contrast to nonsmokers relatives (15.53\%), which are consistent with studies conducted in the USA. Their data reported that number of smoker student's with parents smokers was significantly high $(\mathrm{n}=1178)$ than non-smokers parents $(n=636)$ [22]. Another study from Nigeria showed $27 \%$ of high school non-smoker students with smoker relatives in contrast with $66 \%$ of smoker students with smokers relative out of total $(n=1174)$ [23].

Talking about prevalence from other cities of Saudi Arabia, a study conducted in Riyadh, reported high prevalence of smoking among school-based students which shows the trend of high prevalence in the country. The study also suggests that having low life satisfaction among the smokers [24]. Additional finding of our study was the cross-sectional comparison between students of public and private schools, their smoking behavior, and possible factors for them to smoke. To the best of our knowledge, there are no previous studies comparing schoolchildren of public and private schools. However, a similar study has been performed in Pakistan [25]. Peer attribute of smoking is high in public school $(60 \%)$ as compared to private school students. Higher family education level of private school student's may be the reason as public schoolchildren family lack of education may lead to less knowledge of effects and they are maintaining a restrict environment for their children. Other influential factors which could increase smoking practice in students from public sector could be parents/relatives smoking addiction, smoking in children's presence, low parental education level, no healthy activity, TV/movies role models smoking, and cigarettes smoking promotion on media. Students from private who mostly belong to high socioeconomic group may start smoking due to status symbol, western culture following, school gatherings, ease of affordability, and access [23]. Another study from Saudi Arabia suggested that parents, peer, and siblings were the main factor which made students to smoke with additional contributing factors of pocket money, i.e., more than 300 SR per month, and advertisement of cigarette smoking in mass media [26].

\section{Conclusion and Recommendations}

It is concluded from our findings that the prevalence of smoking among children exists in a 
considerable proportion (27\%, who is either smoking or trying to smoke in their early age). Parents and family members' education and awareness of not smoking in front of children, announcement on media about harmful effects, and banning advertisement of cigarette company promotions may help curb the development of smoking habits of our youth.

Based on our study findings, the following are our recommendations:

1. Prevention efforts should be tailored to address children's awareness about both short- and long-term adverse effects of smoking

2. The health and education authorities should take major steps to control smoking within school premises

3. More large-scale multicenter studies are needed to estimate the accurate prevalence of smoking among schoolchildren.

\section{Limitation of the study}

This study has few weaknesses. Even though this questionnaire is used in many studies to estimate prevalence, it still lacks few aspects that may be the contributing factors making children smoke such as having other family members who smoke. Therefore, some modifications were made to tailor the questions considering Saudi culture. Another limitation was the number of students recruited from private and public schools ( $n=660$ vs. 223), ideally, it should be approximately similar number of students in each group. This may be because private schools only have limited seats for each class and have stricter policies to participate in research studies than public schools.

\section{References}

1. Guindon GE, Boisclair D. Past, Current and Future Trends in Tobacco Use. Washington, DC: Health, Nutrition and Population Discussion Paper \#6, World Bank; 2003.

2. Samet JM. Tobacco smoking: The leading cause of preventable disease worldwide. Thorac Surg Clin. 2013;23(2):103-12.

\section{PMid:23566962}

3. Hefler M, Liberato SC, Thomas DP. Incentives for preventing smoking in children and adolescents. Cochrane Database Syst Rev. 2017;6(6):CD008645. https://doi.org/10.1002/14651858. cd008645.pub3

PMid:28585288

4. White VM, Bariola E. Australian Secondary School Students Use of Tobacco, Alcohol, and Over-the-counter and Illicit Substances in 2011: Report. Australia: National Drug Strategy, Department of Health and Ageing; 2012.

5. Fida HR, Abdelmoneim I. Prevalence of smoking among male secondary school students in Jeddah, Saudi Arabia. J Family Community Med. 2013;20(3):168. https://doi. org/10.4103/2230-8229.121993

\section{PMid:24672274}

6. Hasim TJ. Smoking habits of students in college of applied medical science, Saudi Arabia. Saudi Med J. 2000;21(1):76-80. PMid:11533755

7. Al Ghobain MO, Al Moamary MS, Al Shehri SN, AL-Hajjaj MS. Prevalence and characteristics of cigarette smoking among 16 to 18 years old boys and girls in Saudi Arabia. Ann Thorac Med. 2011;6(3):137. https://doi.org/10.4103/1817-1737.82447 PMid:21760845

8. Al Moamary MS, Al Ghobain MO, AI Shehri SN, Gasmelseed AY Al-Hajjaj MS. Predicting tobacco use among high school students by using the global youth tobacco survey in Riyadh, Saudi Arabia. Ann Thorac Med. 2012;7(3):122. https://doi. org/10.4103/1817-1737.98843

PMid:22924068

9. Sadeghi R, Mahmoodabad SS, Fallahzadeh H, Rezaeian M, Bidaki R, Khanjani N. Predictive factors for preventing hookah smoking and health promotion among young people based on the protection motivation theory. J Educ Health Promot. 2019;8:169. https://doi.org/10.1093/heapro/daz109 PMid:31867354

10. Martinasek MP, McDermott RJ, Martini L. Waterpipe (hookah) tobacco smoking among youth. Curr Probl Pediatr Adolesc Health Care. 2011;41(2):34-57. https://doi.org/10.1016/j. cppeds.2010.10.001

PMid:21232693

11. Pirdehghan A, Aghakoochak A, Vakili M, Poorrezaee $M$. Determination of predicting factors of Hookah smoking among pre-university students in Yazd in 2015. Pajouhan Sci J. 2016;15(1):28-36 .

12. Hopkinson NS, Lester-George A, Ormiston-Smith N, Cox A, Arnott D. Child uptake of smoking by area across the UK. Thorax. 2014;69(9):873-5. https://doi.org/10.1136/ thoraxjnl-2013-204379

PMid:24304854

13. El Mhamdi S, Wolfcarius-Khiari G, Mhalla S, Salem KB, Soltan $\mathrm{SM}$. Prevalence and predictors of smoking among adolescent schoolchildren in Monastir, Tunisia. East Mediterr Health J. 2011;17(6):523. https://doi.org/10.26719/2011.17.6.523 PMid:21796971

14. Norbanee TH, Norhayati MN, Norsa'adah B, Naing NN Prevalence and factors influencing smoking amongst Malay primary school children in Tumpat, Kelantan. Southeast Asian J Trop Med Public Health. 2006;37(1):230.

PMid:16771239

15. Lemeshow S, Hosmer DW Jr. A review of goodness of fit statistics for use in the development of logistic regression models. Am J Epidemiol. 1982;115(1):92-106. https://doi. org/10.1093/oxfordjournals.aje.a113284 PMid:7055134

16. Abuse S. Results from the 2005 National Survey on Drug Use and Health: National Findings. Available from: https://www.dpft. org/resources/NSDUHresults2005.pdf. [Last accessed on 2020 Jan 05]

17. Wong LP, Alias H, Aghamohammadi N, Aghazadeh S, Hoe VC Shisha smoking practices, use reasons, attitudes, health effects and intentions to quit among shisha smokers in Malaysia. Int J Environ Res Public Health. 2016;13(7):726. https://doi. org/10.3390/ijerph13070726

PMid:27447655

18. American Cancer Society. Cancer Facts and Figures 2013. Atlanta, GA: American Cancer Society; 2013.

19. U.S. Department of Health and Human Services. The Health Consequences of Smoking-50 Years of Progress: A Report of the Surgeon General. Atlanta: U.S. Department of Health 
and Human Services, Centers for Disease Control and Prevention, National Center for Chronic Disease Prevention and Health Promotion, Office on Smoking and Health; 2014. Available from: Available at https://aahb.org/Resources/ Pictures/Meetings/2014-Charleston/PPT\%20Presentations/ Sunday\%20Welcome/Abrams.AAHB.3.13.v1.o.pdf. [Last accessed on 2019 Jun 15]. https://doi.org/10.1037/ e300842003-001

20. U.S. Department of Health and Human Services. Preventing Tobacco Use Among Youth and Young Adults: A Report of the Surgeon General. Atlanta, GA: U.S. Department of Health and Human Services, Centers for Disease Control and Prevention, National Center for Chronic Disease Prevention and Health Promotion, Office on Smoking and Health; 2012. p. 3. https:// doi.org/10.1037/e300842003-001

21. Coleman BN, Johnson SE, Tessman GK, Tworek C, Alexander J, Dickinson DM, et al. It's not smoke. It's not tar. It's not 4000 chemicals. Case closed: Exploring attitudes, beliefs, and perceived social norms of e-cigarette use among adult users. Drug Alcohol Depend. 2016;159:80-5. https://doi.org/10.1016/j. drugalcdep.2015.11.028

\section{PMid:26708706}

22. Fakhari A, Mohammadpoorasl A, Nedjat S, Hosseini MS, Fotouhi A. Hookah smoking in high school students and its determinants in Iran: A longitudinal study. Am J Mens Health 2015;9(3):186-92. https://doi.org/10.1177/1557988314535236 PMid:24855098

23. Gilman SE, Rende R, Boergers J, Abrams DB, Buka SL, Clark MA, et al. Parental smoking and adolescent smoking initiation: An intergenerational perspective on tobacco control. Pediatrics. 2009;123(2):e274-81. https://doi.org/10.1542/ peds.2008-2251 PMid: 19171580

24. Alsubaie AS. Prevalence and determinants of smoking behavior among male school adolescents in Saudi Arabia. Int J Adolesc Med Health. 2018. DOI: 10.1515/ijamh-2017-0180. PMid:29369814

25. Rozi S, Akhtar S, Sajid A, Khan J. Prevalence and factors associated with current smoking among high school adolescents in Karachi, Pakistan. Southeast Asian J Trop Med Public Health. 2005;36(2):498.

PMid:15916062

26. Al-Zalabani A, Kasim K. Prevalence and predictors of adolescents' cigarette smoking in Madinah, Saudi Arabia: A school-based cross-sectional study. BMC Public Health. 2015;15(1):17. https://doi.org/10.1186/s12889-015-1363-8 PMid:25604704 